\title{
Research on software trustworthiness measurement evaluation model based on data driven
}

\author{
Zhaosheng $\mathrm{Xu}^{*}$ \\ College of Mathematics and Computer, Xinyu University, Xinyu 338000, China
}

\begin{abstract}
. in this paper, we construct a data-driven trust evaluation model based on perceptual source. The model takes the monitoring module as the evaluation unit, and the relay node completes the trust evaluation of the sensing node in its monitoring module. The direct trust calculation is realized by the relationship between the sensing node's own data, and the recommended trust is calculated by using the relationship between the neighbor nodes in the monitoring module. Combined with the historical trust, the comprehensive trust of the sensing node is output. In this paper, the credibility of existing database driven cognitive software is summarized, and then the key technologies of reliability evaluation and classification of database driven cognitive software are analyzed.
\end{abstract}

\section{Preface}

Wireless sensor network is an important form of the perception layer of the Internet of things. The sensor nodes of wireless sensor network are usually used for data collection in different network environments and applications, and can master the status information of monitoring area in real time, so that more and more physical entities are connected to the Internet through sensors. Based on the concept of dishonest evidence, this paper proposes a comprehensive software trustworthiness measurement method, and introduces a data-driven software trustworthiness measurement evaluation model in detail. Through the large-scale deployment of wireless sensor networks in the sensing layer, the original data collected by the sensors are obtained, and then transmitted to the gateway by using the self-organizing network protocol, which makes it become a data centric common Enjoy the Internet. Hope to provide a reference for the development of related technologies.

\section{Introduction of database driven cognitive software credibility}

The credibility of database driven cognitive software mainly refers to the way to obtain the credibility. The available modeling method is to locate its own reliability and obtain it after querying the database of its own reliability. Because the credibility of traditional cognitive software can not be obtained by other means, some modeling is very crowded and some

\footnotetext{
*Corresponding author: 40393574@qq.com
} 
modeling is idle. Updating the software credibility mechanism through the relevant database can not only expand the scope of cognitive reliability modeling perception ability, but also enable the software credibility without modeling perception ability to obtain the corresponding ability through the relevant database channel. It is not only convenient for managers to adjust and control, but also can make the credibility clear the scope of application of their own modeling.

The database of database driven cognitive software credibility is based on related database, which is composed of DB (database), PU (authorization trust) and su (cognitive reliability). The workflow can be divided into preparation stage (Su obtains the uniform resource identifier of DB), and inquiry stage (Su sends device descriptor, device descriptor, related location and master device related information to DB) Information and other parameters), reply stage (DB authenticates and replies to Su's request to confirm whether $\mathrm{Su}$ is within the valid range of database), feedback stage (DB can use Su's request to complete modeling after DB completes reply). The credibility of database driven cognitive software is based on the white space database access protocol (paws) and uses HTTPS as the transmission mechanism. At the same time, it also exposes the problems of privacy and accuracy. In order to minimize the impact of these two aspects, the credibility of database driven cognitive software needs to ensure the following four points: first, to ensure the effective number of database use and query According to the database; secondly, the information to be transmitted needs to be encrypted; thirdly, the connection mode is confirmed to be correct; finally, the transmission message ensures the formality of its content.

\section{Reliability evaluation and classification of database driven cognitive software}

At the beginning of modeling, the sensing area of the sensing layer is divided into several monitoring modules. The sensing nodes in each monitoring module complete the same monitoring task and belong to the same relay node. Because only the trust evaluation of the sensing nodes is analyzed, it is assumed that the relay nodes deployed in the system are reliable. The sensor node only collects a certain indicator data and sends it to the relay node by short-range communication technology, and does not forward the data of other nodes; the relay node is only responsible for fusing and forwarding the sensing data, and calculating the trust degree of each sensing node in its monitoring module, filtering the data collected by the sensing node, and finally transmitting it to the sink node.

According to the author's review and collation of relevant literature and materials, the author thinks that the credibility evaluation and classification of database driven cognitive software can be divided into two aspects: perception node and weighted calculation.

\subsection{Sensing node}

The node feedback itself is easy to be tampered and interfered by the outside world, so the credibility model of database driven cognitive software based on node feedback naturally has the disadvantage of being easily tampered and interfered by the outside world. When the credibility of database driven cognitive software is carried out, the relevant cognitive reliability is easy to be affected by the outside world and obtain the misplaced position information, thus hindering cognition The reliability can be modeled correctly, so the perception node has become an unavoidable problem in the credibility of database driven cognitive software. To solve this problem fundamentally, it is necessary to set 
corresponding recognition and error correction capabilities according to the characteristics of database driven cognitive software credibility.

\subsection{Weighted calculation}

Due to the redundancy of the sensor nodes in the Internet of things oriented wireless sensor network, the sensing data of the sensing layer has the characteristics of continuity and non hopping. Taking the monitoring module as the evaluation unit, the nodes in the same monitoring module are neighbors to each other. According to the characteristics of the actual application scenarios of the Internet of things, for the monitoring of the same indicators, the data values perceived by the sensing nodes in the same monitoring module are similar, which can realize the trust evaluation between the sensing nodes themselves and other sensing nodes in the same monitoring module. The comprehensive trust evaluation of the output of this model consists of three parts: direct trust, recommendation trust and historical trust. The evaluation of direct trust is the aggregation of the real-time data and historical data of the active sensing node itself for the sensing node itself; the recommended trust is generated between the sensing nodes, which is the aggregation of a single active sensing node and other active sensing nodes in the monitoring module; the initial value of historical trust is set by human, and is constantly updated with the operation of the network. Finally, the comprehensive trust of the sensing node is obtained by the weighted calculation of the three trust values. Then, the state of the sensing node is judged by comparing with the set suspicious threshold and abnormal threshold, and the historical trust and trust list are updated. This process can realize the anomaly detection of the sensing node.

According to the above analysis, the weighted calculation of reliability can be divided into two cases, one is direct weighted calculation, the other is indirect weighting calculation. As the name implies, direct weighted calculation is to infer the trust feedback through the available modeling of the reliability of the location information provided by the reliability; while the indirect weighting calculation is to infer the use of the trust modeling through other methods, and after multiple rounds of switching, the relevant reliability can be locked to a minimum range.

\section{Summary}

In a word, the trust mechanism is introduced to participate in the data fusion process, and the comprehensive trust is taken as the fusion parameter, and the influence of trust evaluation model on data fusion is compared and analyzed. The results show that the fusion data with trust evaluation is more accurate no matter whether the network is in the normal state or abnormal nodes. Because the model eliminates the abnormal nodes in time, the abnormal conditions in the monitoring module are improved and the stability of data fusion is improved. I hope that the author will have the opportunity to continue to study related issues in the future, and further improve the credibility evaluation of data-driven cognitive software on the basis of ensuring the follow-up work.

\section{References}

1. Wu Lijin, Han Xinyu, Yan Ran, Xu Zhaowei, Tang Longli. Research on reliability analysis and design technology of ship equipment software $[\mathrm{J}]$. Computer measurement and control, 2018,26 (12): 130-135+ 170 
2. Liu miaochen. Research and application of rest style software credibility test based on behavior statement [D]. Beijing University of technology, 2017

3. Zhang Weixiang, Liu Wenhong, Wu Xin. Quantitative evaluation method of software trustworthiness based on data fusion [J]. Computer science, 2013,40 (S2): 192-195 + 213 